\title{
Mineral plastic hydrogels from the cross-linking of polyacrylic acid and alkaline earth or transition metal ions
}

\author{
David J. Schupp, ${ }^{a}$ Xiaotong Zhang, ${ }^{b}$ Shengtong Sun ${ }^{\star b}$ and Helmut Cölfen (ID*a
}

\begin{abstract}
Used polymer materials generate huge environmental problems nowadays. A new solution was recently provided by a physically cross-linked polyacrylic acid-based mineral plastic hydrogel, which is resistant in the anhydrous state and easily recyclable. Here we report that substituting the cross-linker by various cations and controlling the metal content can provide promising materials with analogous properties.
\end{abstract}

Over 300 million tons of plastics are produced per year. ${ }^{1}$ Plastics combine several advantageous properties, e.g. they are lightweight, strong, and cheap with cost-efficient formability. ${ }^{2}$ Besides, plastic materials are good insulators for heat or electric current, flexible, chemically stable and can exhibit controllable properties. ${ }^{3}$ For example, commercial plastics such as polyethylene (LDPE, LLDPE, MDPE and HDPE), polypropylene (PP) and polyethylene terephthalate (PET) are mostly used in both daily life and the industrial field. ${ }^{1}$ However, due to their high stability under ambient conditions ${ }^{4-6}$ - numerous conventional plastics decompose only within several centuries ${ }^{7,8}$ - post-consumer plastics have already generated serious environmental problems. ${ }^{9-13}$ Used plastics accumulate especially in the oceans and form garbage patches or end up as so-called micro plastics with uncertain consequences for nature and humanity. ${ }^{14,15}$

To address this issue, a feasible solution is to find energy-efficient and recyclable substitute plastic materials. Inspired by the polymerinduced liquid precursor phase in the bio-mineralization process, ${ }^{16}$ we have recently reported a new kind of organic-inorganic hybrid material, called 'mineral plastic', which consists of polyacrylic acid (PAA, $M_{\mathrm{w}} \approx 100000 \mathrm{~g} \mathrm{~mol}^{-1}$ ) non-covalently cross-linked via amorphous calcium carbonate (ACC) nanoparticles. ${ }^{17,18}$

\footnotetext{
${ }^{a}$ Physical Chemistry, Department of Chemistry, University of Konstanz, Universitätsstrasse 10, 78457 Konstanz, Germany.

E-mail: helmut.coelfen@uni-konstanz.de

${ }^{b}$ Center for Advanced Low-dimension Materials, Donghua University,

Shanghai 201620, China. E-mail: shengtongsun@dhu.edu.cn
}

The synthetic procedure of an ACC-PAA hydrogel is very simple by just adding sodium carbonate into a solution of calcium chloride and PAA, forming a shapeable, stretchable, and selfhealable gel. ${ }^{17}$ Upon drying in air, the hydrogel gradually becomes a transparent and rigid object, and this process is totally reversible upon immersing in water again, indicating the good recyclability of this material. Owing to these unique properties, the ACC-PAA mineral plastic hydrogel has been successfully applied in capacitive ionic skin sensors, ${ }^{19}$ multifunctional graphene composites, ${ }^{20}$ and organic-inorganic hybrid adhesives. ${ }^{21}$ These results strongly indicate the potential of this material but a question remains if the basis for mineral plastics can be extended.

Here we show that similar mineral plastic-like hydrogels and rigid hybrid materials can also be produced from the substitution of $\mathrm{Ca}^{2+}$ by other alkaline earth and transition metal ions such as $\mathrm{Mg}^{2+}, \mathrm{Sr}^{2+}, \mathrm{Ba}^{2+}, \mathrm{Mn}^{2+}, \mathrm{Ni}^{2+}$ and $\mathrm{Zn}^{2+}$. We also find that it is crucial for the hydrogel formation to control the deprotonation degree of the carboxylic acid groups of PAA and the metal content.

In a typical experiment, $0.1 \mathrm{M}$ sodium carbonate solution was added into a mixed solution of $0.1 \mathrm{M}$ PAA $\left(M_{\mathrm{w}} \approx 100000 \mathrm{~g} \mathrm{~mol}^{-1}\right)$ and respective $0.1 \mathrm{M}$ metal chloride, gradually forming a plastic and self-healable hydrogel around the stirring bar accompanied by a turbid solution containing non-gelling fractions (see experimental details in ESI $\dagger$ ). The swollen hydrogels are easily processible due to their separation from the aqueous phase. The $\mathrm{pH}$ value of the reaction mixture was adjusted by the amount of sodium carbonate added and was between 2.8 and 7.5 (see Table 1). At most of the preparation $\mathrm{pH}$, the amount of hydroxide and carbonate in the final materials is negligible. A thorough mixing and slow addition rate of sodium carbonate (otherwise stated, an addition rate $\sim 3 \mathrm{ml} \mathrm{min}^{-1}$ was chosen for all the hydrogels) is important for the hydrogel formation. The speed of base addition seems to affect the properties of final materials significantly (Fig. S2.3, ESI $\uparrow$ ), and further investigations regarding the controlled base-addition and stirring speed are required to determine their effects on the resulting materials. 
Table 1 Summary of $\mathrm{pH}$ values for hydrogel formation and metal contents of all the mineral plastics. The metal contents of the dried hybrids are given in atomic/at\% and weight percent/wt\% estimated via EDX and TGA

\begin{tabular}{lllll}
\hline & & $\begin{array}{l}\mathrm{M}^{2+} / \mathrm{at} \% \\
(\mathrm{EDX})\end{array}$ & $\begin{array}{l}\mathrm{M}^{2+} / \mathrm{wt} \% \\
(\mathrm{EDX})\end{array}$ & $\begin{array}{l}\mathrm{M}^{2+} / \mathrm{wt} \% \\
(\mathrm{TGA})^{b}\end{array}$ \\
\hline Mg-PAA & 7.5 & 5.1 & 8 & 12 \\
Ca-PAA & 7.0 & 5.3 & 14 & 15 \\
Sr-PAA 1 & 7.2 & 4.8 & 24 & 26 \\
Sr-PAA 2 & 4.6 & 3.1 & 17 & 19 \\
Ba-PAA & 2.8 & 2.9 & 20 & 21 \\
Ni-PAA & 6.3 & 6.0 & 21 & 20 \\
Mn-PAA & 6.4 & 6.2 & 21 & $19-22$ \\
Zn-PAA & 6.2 & 6.3 & 23 & 21
\end{tabular}

${ }^{a} \mathrm{pH}$ values with maximum metal contents in the respective hydrogels (except for Sr-PAA 2). ${ }^{b}$ The metal content was estimated via the amount of formed metal oxide during pyrolysis (Fig. S2.2, ESI).

Similar to the ACC-PAA hydrogel in the case of $\mathrm{Ca}^{2+}$, the resulting hydrogels of Sr-PAA, Ni-PAA, Mn-PAA and Zn-PAA exhibit pastelike plasticity and instant self-healing in the swollen state and can reversibly form transparent, free-standing, and macroscopically continuous films in the anhydrous state (Fig. 1 and 2). The excellent self-healing ability of the gels within minutes originates from the dynamic physical cross-linking of PAA chains (Fig. 2). The transformation between the swollen and dried states is totally reversible and even immersing the hydrogel in water for over 20 days does not affect this process. It is noted that the green color of the $\mathrm{Ni}-\mathrm{PAA}$ hybrid arises from the $\mathrm{Ni}^{2+}-\mathrm{COO}^{-}$complexes. The $\mathrm{Mg}_{-}$ PAA hydrogel is unstable in a vigorously stirred solution due to a decreased cross-linking ability leading to a viscous gel instead of a plastic hydrogel and it is thus not shown in Fig. 1. The Ba-PAA hydrogel only precipitates at a remarkably low pH of 2.8 (Table 1), whereas a higher $\mathrm{pH}$ produces non-swellable agglomerates. Besides, the Ba-PAA hydrogel does not self-heal within minutes, suggesting a degraded plasticity, and its hybrid is also not as transparent as other M-PAA hybrids after drying (Fig. 1). This indicates that the formation and properties of M-PAA hydrogels strongly depend on the type of used cations.

The presence and percentage of the respective metal atoms in the hybrids were verified and calculated by EDX and TGA, which gave very similar results (Fig. S2.1, S2.2, ESI $\dagger$ and Table 1). Note that TGA measurements of the dried M-PAA gels may show an over-estimated metal carbonate content, as the complex of $\mathrm{M}^{2+}$ and carboxylate could lead to an instant

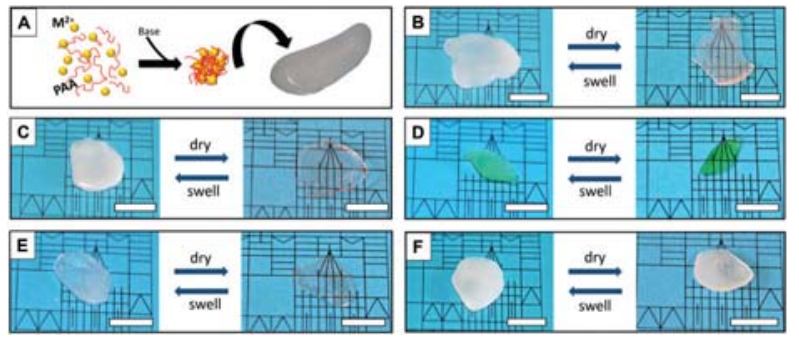

Fig. 1 Proposed formation mechanism (A) and appearance of all mineral plastic hydrogels in the swollen and dried states: (B) Sr-PAA, (C) Mn-PAA (D) Ni-PAA (E) Zn-PAA and (F) Ba-PAA. Scale bar $=1 \mathrm{~cm}$.

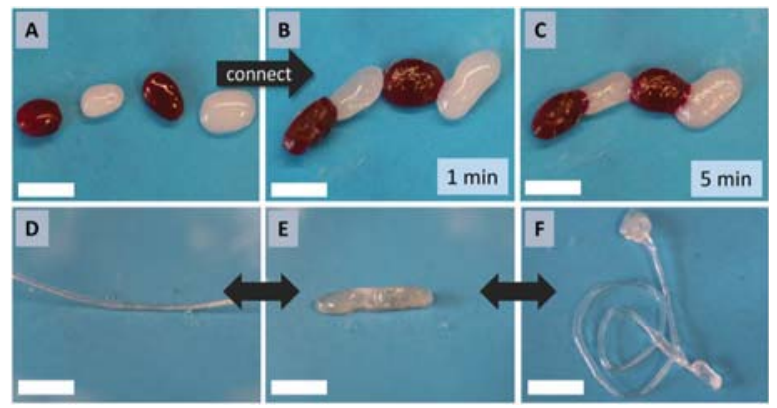

Fig. 2 The hydrogels show excellent self-healing ability due to the dynamic physical cross-linking. (A) Separated Mn-PAA hydrogels in the swollen state. The dark red color results from the addition of Toluylene red. ( $B$ and $C$ ) Upon connection, the gels heal themselves within minutes. (D-F) The Mn-PAA hydrogel is highly plastic with various deformed shapes. Scale bar $=1 \mathrm{~cm}$

formation of metal carbonate during pyrolysis, if the deposition temperature is higher than that of PAA itself. ${ }^{22}$ The IR spectra of M-PAA hydrogels reveal the deprotonation of most of the $\mathrm{COOH}$ groups of PAA except Ba-PAA, suggesting the formation of $\mathrm{M}^{2+}-\mathrm{COO}^{-}$complexes upon the addition of $\mathrm{Na}_{2} \mathrm{CO}_{3}$ (Fig. 3). Interestingly, judging from the peak intensities of $\nu(\mathrm{COOH})$ around $1700 \mathrm{~cm}^{-1}$ which is mainly influenced by the used $\mathrm{pH}$ of the reaction mixture (Table 1), the deprotonation degree of PAA for the maximum alkaline earth metal content increases with the decrease in the atomic radius, in an order of Ba-PAA, Sr-PAA, Ca-PAA, and Mg-PAA (Fig. 3). This order is consistent with the observation on the hydrogel formation, suggesting that the degree of deprotonated carboxyl groups is crucial for making mineral plastic hydrogels. This becomes even clearer when we prepare Sr-PAA hydrogels at $\mathrm{pH} 7.2$ and 4.6 (Table 1) and Mn-PAA hydrogels at $\mathrm{pH} 6.4$ and 4.4. As a supplement to TGA, the Mn content was determined by AAS measurements, which showed a Mn content of $20 \mathrm{wt} \%$ at $\mathrm{pH} 6.4$ and $16 \mathrm{wt} \%$ at $\mathrm{pH}$ 4.4, respectively. A higher $\mathrm{pH}$ leads to a higher metal content included in the hybrid.

The findings suggest that there is a maximum metal content for each cation/hydrogel, which was also observed for ACC-PAA synthesized at different concentrations. ${ }^{17}$ An explanation for the observed order of the deprotonation degree within the

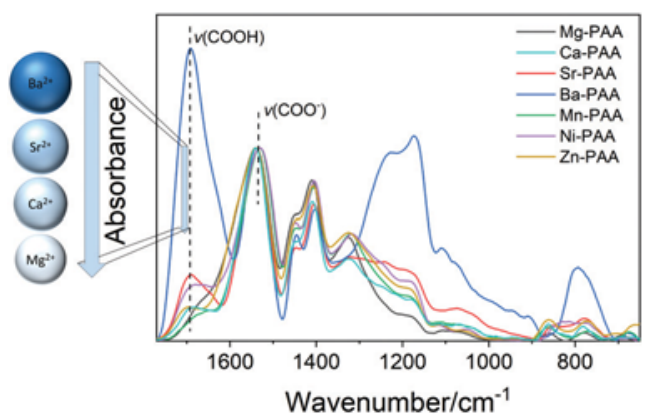

Fig. 3 The ATR-FTIR spectra of all mineral plastics indicate the complexation between carboxylates and respective metal ions (around $1530 \mathrm{~cm}^{-1}$ ) and the deprotonation degree of PAA (around $1700 \mathrm{~cm}^{-1}$ ). 
formed hydrogels could be provided by the HSAB concept of binding. Softer acids like $\mathrm{Ni}^{2+}, \mathrm{Sr}^{2+}$ or $\mathrm{Ba}^{2+}$ favor a decreased deprotonation degree whereas hard acids like $\mathrm{Ca}^{2+}, \mathrm{Mn}^{2+}$ and $\mathrm{Mg}^{2+}$ prefer an increased deprotonation of PAA. A comparison of the atomic content of particular metal ions (Table 1) indicates a slightly increased metal content in hydrogels using transition metals instead of alkaline earth metals. In addition, the respective maximum metal content occurs consistently at lower $\mathrm{pH}$ values for transition metal hydrogels compared to that for alkaline earth metals, which indicates a stronger binding ability of the transition metal ions with deprotonated PAA chains than that of the alkaline earth metal ions. These findings are consistent with the literature. ${ }^{23}$ Therefore, regarding the nature and strength of crosslinking, it has to be distinguished between the alkaline earth and transition metals. In contrast to the ionic interactions of alkaline earth metals, some transition metals like $\mathrm{Cu}$ and $\mathrm{Fe}$ show an enhanced covalent character for the metal-oxygen bond. ${ }^{23}$ Therefore, employment of $\mathrm{Cu}^{2+}$ and $\mathrm{Fe}^{2+}$ results in the formation of nonplastic agglomerates. In the other studies, trivalent cations were used to synthesize polyacrylic acid-based hydrogels. ${ }^{24,25}$ As we tested, the usage of $\mathrm{Al}^{3+}$ and $\mathrm{Fe}^{3+}$ results in non-plastic and nonself-healing precipitates (Fig. S2.5, ESI $\dagger$ ). Trivalent cations possibly exhibit too strong $\mathrm{M}^{3+}-\mathrm{COO}^{-}$interactions leading to a rigid and non-self-healing M-PAA network. Concluding from the formation of hydrogels, a dependence between the atomic radius of used alkaline earth metals and the binding affinity with PAA seems plausible. This may explain the formation of an unstable Mg-PAA hydrogel due to a weak intermolecular crosslinking, whereas too strong crosslinking causes a decreased plasticity for Ba-PAA.

Rheology measurements of the swollen hydrogels reveal an analogous behaviour to the already documented ACC-PAA hydrogels (Fig. S2.3, ESI $\dagger$ ). ${ }^{17}$ All the analysed hydrogels show viscoelasticity strongly depending on the angular frequency, which is typical of physically cross-linked gels. ${ }^{17}$ The semiliquid state of the hydrogels is indicated by the almost equal loss $\left(G^{\prime \prime}\right)$ and storage moduli $\left(G^{\prime}\right)$. In comparison to the other hydrogels $(<1000 \mathrm{~Pa}),{ }^{26}$ all the M-PAA hydrogels exhibit higher values for $G^{\prime}$ and $G^{\prime \prime}\left(10^{3}-10^{5} \mathrm{~Pa}\right)$. In addition, the swollen gels exhibit a shear-thinning behaviour, which is a typical property of pseudoplastic fluids ${ }^{27}$ and therefore explains their good shapeability and plasticity. ${ }^{17}$ Nanoindentation measurements of the dried gels of thin M-PAA hybrid films revealed a strong decrease in their hardness and modulus by exchanging alkaline earth metals with bivalent metal ions (Fig. S2.4, ESI $\dagger$ ).

Given the important role of the deprotonation degree of PAA, the synthesis of M-PAA hydrogels without the usage of sodium carbonate also seems feasible. Indeed, as we tested for calcium and strontium, hydrogels with analogous properties like ACC-PAA are accessible without carbonates, too. By increasing the $\mathrm{pH}$ of a reaction mixture of PAA $\left(M_{\mathrm{w}} \approx 100000 \mathrm{~g} \mathrm{~mol}^{-1}\right)$ and metal chloride using sodium hydroxide solution (under an inert atmosphere to exclude carbonate development from atmospheric $\mathrm{CO}_{2}$ ), mineral plastic-like hydrogels were also obtained. The hydrogel simply consists of PAA chains connected via
$\mathrm{Ca}^{2+}$ or $\mathrm{Sr}^{2+}$, sort of a water-insoluble Ca-PAA or Sr-PAA salt. The rheological results of the Ca-PAA hydrogel reveal a much lower modulus than that of the ACC-PAA hydrogel (Fig. S2.3, ESI $\dagger$ ), probably due to a decreased cohesion strength with a lower crosslinking density when no carbonates are included. Nevertheless, this experiment clearly indicates that the crosslinking between deprotonated PAA chains and $\mathrm{Ca}^{2+}$ should be the main reason for the formation of the ACC-PAA hydrogel in the case of $\mathrm{Na}_{2} \mathrm{CO}_{3}$.

In conclusion, we show in this paper that ACC-PAA like mineral plastic hydrogels can also be easily achieved from the substitution of $\mathrm{Ca}^{2+}$ with different bivalent cations and even without the addition of carbonates leading to the mineral plastic previously described by us. ${ }^{17}$ We highlight the crucial role of crosslinking between metal ions and PAA chains in the hydrogel formation, which is reflected by the proper deprotonation degree of PAA (depending on $\mathrm{pH}$ ) and the metal content. The present new possibilities of synthesizing mineral plastic materials are highly promising for various commercial applications due to their simplicity and adjustable and outstanding properties accompanied by a cost-efficient synthesis.

S. T. S. gratefully acknowledges the financial support from the National Natural Science Foundation of China (No. 51873035, 21604024). The authors thank Ann-Kathrin Göppert for TGA and Meng Tian of Fudan University for nanoindentation measurements.

\section{Conflicts of interest}

There are no conflicts to declare.

\section{Notes and references}

1 PlasticsEurope (PEMRG)/Consultic (Plastics - the Facts 2017), https:/www.plasticseurope.org/application/files/5715/1717/4180/ Plastics_the_facts_2017_FINAL_for_website_one_page.pdf, accessed October 2018.

2 C. Vasile, Handbook of polyolefins synthesis and properties, Marcel Dekker Inc., New York, 1993.

3 R. Young and P. Lovell, Introduction to Polymers, 3rd edn, CRC Press, Boca Raton, 2011.

4 R. J. Müller, Proc. Biochem., 2006, 41, 2124-2128.

5 D. W. Laist, Mar. Pollut. Bull., 1987, 18, 319-326.

6 J.-D. Gu, Int. Biodeterior. Biodegrad., 2003, 52, 69-91.

7 P. G. Ryan and C. L. Moloney, Nature, 1993, 361, 23.

8 J. G. B. Derraik, Mar. Pollut. Bull., 2002, 44, 842-852.

9 C. J. Moore, Environ. Res., 2008, 108, 131-139.

10 A. A. Shah, F. Hasan, A. Hameed and S. Ahmed, Biotechnol. Adv., 2008, 26, 246-265.

11 M. R. W. Rands, W. M. Adams, L. Bennun, S. H. M. Butchart, A. Clements, D. Coomes, A. Entwistle, I. Hodge, V. Kapos, P. W. Scharlemann Jr., W. J. Sutherland and B. Vira, Science, 2010, 329, 1298-1303.

12 D. K. Barnes and P. Milner, Mar. Biol., 2005, 146, 815-825.

13 P. G. Ryan, C. J. Moore, J. A. van Franeker and C. L. Moloney, Philos. Trans. R. Soc., B, 2009, 364, 1999-2012.

14 M. Cole, P. Lindeque, C. Halsband and T. S. Galloway, Mar. Pollut. Bull., 2011, 62, 2588-2597.

15 R. C. Thompson, Y. Olsen, R. P. Mitchell, A. Davis, S. J. Rowland, A. W. G. John, D. McGonigle and A. E. Russell, Science, 2004, 304, 838. 16 L. B. Gower, Chem. Rev., 2008, 108, 4551-4627.

17 S. Sun, L. Mao, Z. Lei, S. Yu and H. Cölfen, Angew. Chem., Int. Ed., 2016, 55, 11765-11769.

18 G. Pacchioni, Nat. Rev. Mater., 2016, 1, 16062. 
19 Z. Lei, Q. Wang, S. Sun, W. Zhu and P. Wu, Adv. Mater., 2017, 29, 1700321.

20 S. Lin, Y. Zhong, X. Zhao, T. Sawada, X. Li, W. Lei, M. Wang, T. Serizawa and H. Zhu, Adv. Mater., 2018, 30, 1803004.

21 A. Li, Y. Jia, S. Sun, Y. Xu, B. B. Minsky, M. A. C. Stuart, H. Cölfen, R. von Klitzing and X. Guo, ACS Appl. Mater. Interfaces, 2018, 10, 10471-10479.

22 I. C. McNeill and S. M. T. Sadeghi, Polym. Degrad. Stab., 1990, 30, 267-282.
23 S. Koda, H. Nomura and M. Nagasawa, Biophys. Chem., 1983, 18, 361-367. 24 X. Jiang, N. Xiang, J. Wang, Y. Zhao and L. Hou, Carbohydr. Polym., 2017, 173, 701-706.

25 Z. Wei, J. He, T. Liang, H. Oh, J. Athas, Z. Tong, C. Wang and Z. Nie, Polym. Chem., 2013, 4, 4601-4605.

26 L. Bromberg, M. Temchenko, V. Alakhov and T. A. Hatton, Int. J. Pharm., 2004, 282, 45-60.

27 K. Baezly, Rheometry: Industrial Applications, Research Studies Press, Chichester, 1980. 